\title{
SYSTEMIC LUPUS ERYTHEMATOSUS AND VIRUS C INFECTION - DIAGNOSIS, EVOLUTION AND TREATMENT PARTICULARITIES
}

\author{
Delia-Georgiana Tudorovici (Rotariu), Mircea Penescu \\ "Dr. Carol Davila" Clinical Hospital of Nephrology, Bucharest \\ Corresponding address \\ rotariu.delia.georgiana@gmail.com
}

\begin{abstract}
Lupus erythematosus (SLE) is an autoimmune disease with a broad spectrum of clinical and immunologic manifestations. Hepatitis C virus (HCV) has been postulated as a potential etiologic or triggering agent among other viruses and it appears to be associated with the presence of autoimmune disorders, even mimicking SLE clinically and serologically. Data on the association between HCV infection and SLE are scarce, but an interaction between these two conditions seems possible.

As for treatment options, it is well known that the classic antiviral therapy with interferon may aggravate preexisting autoimmunity, unmask previously silent autoimmune processes, or even cause the emergence of de novo autoimmune disease, including SLE. Interferon-free treatment seems to be more efficacious, safer and more tolerable. However, it is important that clinicians be aware that DAAs (direct acting antivirals) can also trigger lupus-like immune complex-mediated glomerulonephritis.

There is scant data on the use of immunosuppressive drug therapy in HCV patients. It seems that enhanced HCV replication due to immunosuppression does not lead to clinically significant sequelae and growing evidence has been reported that supports its efficacy and safety.

Patients with SLE and virus C infection are a special category, a fact that the clinician needs to take in consideration in order to a better approach, all the more as these patients are at increased risk for developing end-stage renal disease and have a lower survival rate than general population.
\end{abstract}

Keywords: lupus erythematosus, hepatitis C virus, interferon, antivirals, immunosuppressi 


\section{INTERNAL}

\section{General Reviews}

\section{Rezumat}

Lupusul eritematos sistemic este o afecțiune autoimună cu un spectru larg de manifestări clinice şi imunologice. Printre posibilele cauze sau triggeri ai lupusului a fost incriminată şi infecția cu virusul hepatitic C care se poate asocia cu fenomene autoimune, putând chiar mima clinic şi serologic lupusul.

Opțiunile de tratament includ terapia antivirală şi imunosupresia, dar terapia clasică antivirală cu interferon agravează autoimunitatea, demască fenomene autoimune silențioase sau chiar declanşează boli autoimune de novo, inclusiv lupus. Deşi e mai eficace, fiind mai sigur şi mai uşor de tolerat de pacienți, tratamentul cu noile antivirale cu acțiune directă poate declanşa fenomene autoimune (glomerulonefrită lupus-like). Datele despre imunosupresie în cazul pacienților cu virus $C$ sunt puține în literatura de specialitate, dar s-a constatat că replicarea virală crescută postimunosupresie nu duce la afectare hepatica severă şi tot mai multe studii susțin siguranța şi eficacitatea acesteia.

Pacienții care asociază lupus şi infecție cu virus C reprezintă o categorie aparte. Astfel, clinicianul trebuie să țină cont de faptul că aceştia sunt la risc crescut de a dezvolta boală cronică de rinichi în stadiu final şi au, de obicei, o rată de supraviețuire mai redusă.

Cuvinte cheie: lupus eritematos sistemic, virus hepatitic C, interferon, antivirale, imunosupresie.

SLE (systemic lupus erythematosus) is a complex autoimmune disease with polymorphic clinical manifestations which may affect any system and which has variable evolution. The etiology of this disease is unknown, and is assumed that SLE results from the interaction of immune dysfunction with the genetic background and environmental factors. The viruses, including the $C$ virus, has been postulated as triggering agents in SLE pathogenesis (Table 1, Table 2).

\section{The association between SLE and HCV (hepatitis C virus)}

The study conducted by Ramos-Casals on 134 patients showed a higher prevalence of HCV in patients with SLE compared with general population (11\% vs. $1 \%$ ), supported also by other studies on a larger number of patients ${ }^{(1)}$ (Table 3).

Patients with $C$ virus infection and SLE can be classified into 3 categories: 


\section{Patients with SLE, but false positive Anti-HCV Ab using the ELISA method}

In the study conducted by Cacoub et al., it was possible to confirm Anti-HCV Ab positivity using RIBA-2 in only one patient of the seven patients with SLE and $C$ virus infection confirmed using ELISA-2, and Kowdley et al. showed that only one out of three patients with Anti-HCV Ab detected using ELISA or Imunoblot have $C$ virus infection confirmed by HCV RNA detection using $\mathrm{PCR}^{(4)}$.

\section{Patients with $C$ virus and SLE} confirmed by the presence of Anti-SM $A b$ and of specific lupus manifestations which meet EULAR criteria

Some of the diagnostic criteria for SLE (malar rash, discoid lesions, photosensitivity, neurological manifestations, high ANA and Anti-dsDNA Ab titers, presence of Anti-SM $A b)$ are specific and very rarely encountered only in $\mathrm{C}$ virus infection.

A retrospective study conducted on 19 lupus patients with $C$ virus infection and 42 lupus patients without $C$ virus infection, has established that the prevalence of clinical manifestations of severe lupus as well as serum levels of ANA, Anti-dsDNA $A b$ and complement were similar. However, the prevalence of cryoglobulinemia was higher in patients who associated the 2 comorbidities, with higher ALT levels. The age of onset of SLE was higher in patients with $C$ virus infection, possibly due to extrahepatic manifestations of $C$ virus infection that led to delayed diagnosis of $\mathrm{SLE}^{(5)}$ (Table 4).

Regarding the renal impairment, this may be caused by a C virus associated nephropathy, lupus nephritis or even the combination of the two. Krajewska et al. presents the case of a 25 year old female patient with severe nephrotic syndrome, pancytopenia and retention of nitrogenous wastes syndrome, where immunological and serological tests have revealed positive ANA, elevated AntidsDNA Ab, no cryoglobulinemia, and active infection with genotype $1 \mathrm{C}$ virus.

Renal biopsy revealed class IV lupus nephritis for which immunosuppressive therapy was initiated, with normalized the renal function and the blood cell count. Serum albumin levels and total proteins also increased slightly, with maintaining of nephrotic range proteinuria, despite low immunological activity (Anti-dsDNAAb).

Taking into account the high levels of viral replication, antiviral therapy with ribavirin and interferon was initiated in parallel with oral maintenance corticotherapy, which led to normalization of total serum proteins and of serum albumin and the disappearance of proteinuria.

The evolution of the patient after initiation of antiviral therapy suggests that the autoimmune response to the $C$ virus infection should also be considered ${ }^{(6)}$ (Table 5 ).

\section{Patients with $C$ virus infection} mimicking SLE in presence of low ANA and Anti-dsDNA Ab titer, hypocomplementemia, negative Anti-SM Ab and symptomatology (arthritis, kidney injury)

$C$ virus infection is associated with various autoimmune manifestations mimicking SLE associated symptoms (arthralgia, myalgia, Sicca syndrome) and induces the occurrence of autoantibodies - ANA (20-40\% of HCV p a tients), Anti-ds D N A b, hypocomplementemia that mislead the clinician $^{(1)}$. Seronegative patients (negative 


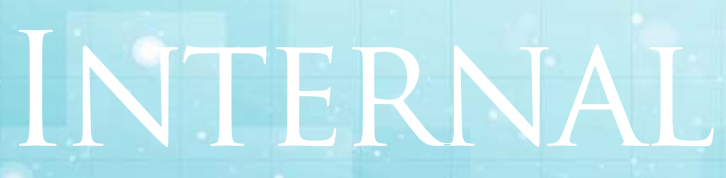

General Reviews

ANA, Anti-dsDNA Ab negative, Anti-SM Ab negative) who have as their first autoimmune disease manifestation a renal manifestation (nephrotic syndrome, nephritic syndrome, etc.) may present fullhouse pattern by immunofluorescence highly suggestive of lupus nephritis. Some of them become seropositive and/or develop extrarenal manifestations of SLE while another category remains seronegative without developing clinical manifestation typical of SLE ${ }^{(7)}$.

Hobeika et al. describe the case of a 53 year old man, diagnosed with chronic $\mathrm{C}$ virus infection 3 years before the onset of kidney impairment. Increased serum creatinine, hypoalbuminemia, hypoproteinemia, proteinuria (13 g/g creatinine) and dysmorphic hematuria were detected at the admission in the clinic of the patient. The serology was negative for ANA, Anti-dsDNA Ab, AntiSSA, AntiSSB, AntiSM, cryoglobulins, and the viral load was $4.5 \mathrm{mil} \mathrm{IU} / \mathrm{mL}$. The renal biopsy revealed cellular crescents and minimal interstitial inflammation and fibrosis, with full-house pattern by immunofluorescence (the presence of IgG, $\lg \mathrm{A}, \lg \mathrm{M}, \mathrm{C} 3$ and $\mathrm{Clq}$ in glomerular basement membrane and mesangial region). The electronic microscopy revealed subepithelial, subendothelial and mesangial immune complexes and tubuloreticular inclusions, which are highly suggestive of lupus nephritis. The final diagnosis was "seronegative lupus" or "lupus-like glomerulonephritis"(8).

\section{Interferon and SLE}

It was found that interferon is capable to exacerbate/unmask silent/pre-existing autoimmune manifestations (autoimmune hepatitis, seronegative arthropathies, rheumatoid arthritis, autoimmune thrombocytopenia) and that patients presenting with low titer of antibodies, associated with $\mathrm{C}$ virus infection, are at higher risk of developing an autoimmune disease, compared to those who do not have detectable antibodies ${ }^{(9)}$.

In patients with SLE, endogenous interferonalpha levels are increased and correlate with disease activity and it has been hypothesized that tubuloreticular inclusion (TRI) morphogenesis in the kidney is related to endogenous (via overproduction) and exogenous (via administration) activity of interferon alpha and beta. Although the incidence of lupus-like syndrome (autoimmune disorders that do not fall within the diagnosis of SLE) is of $0.15-0.7 \%$ to $2 \%$, only a few cases of SLE patients treated with IFN in context of hepatitis $C$ virus occur in the literature. SLE has also been reported in patients following interferon treatment for diseases such as: chronic myeloid leukemia, 
carcinoid tumors, solid tumors, with the onset between 2 weeks and 7 years after the IFN (interferon) therapy initiation ${ }^{(10)}$. The most common manifestations of interferoninduced SLE are arthralgia (most commonly the onset symptom), malar rash, mouth ulcers, photosensitivity and renal impairment (manifestation rarely encountered in druginduced lupus). Immunological changes hypocomplementemia, positive Anti-dsDNA $\mathrm{Ab}$ and Anti-SM Ab, hematologic changes anemia, lymphopenia are associated with these. In most cases, the discontinuation of interferon alpha treatment and a short course of immunosuppression (usually corticotherapy $<6$ months) lead to lupus symptomatology remission and improvement of the immunological parameters ${ }^{(11)}$.

Severe interferon-induced SLE cases are very rare. Vincent Ho et al. present the case of a 43 year old man, following pegIFN and ribavirin treatment for chronic hepatitis $\mathrm{C}$ virus and who after the initiation of antiviral therapy developed maculopapular rash, arthritis and elevated Anti-dsDNA Ab titer. Later, after antiviral therapy discontinuation, the patient met additional criteria of SLE diagnosis: glomerulonephritis, arthritis, serositis, discoid rash, lymphopenia, mouth ulcers, ANA and Anti-dsDNA $A b$ in progressively increased titer. Moreover, he developed myopericarditis and leukocytoclastic vasculitis, lifethreatening complications ${ }^{(12)}$.

\section{DAAs (direct-acting antivirals) and SLE}

The interferon-free therapy acts directly on viral replication and it is considered not to be "immunomodulatory". However, Sise et al. described 3 patients who followed therapeutic regimens with sofosbuvir and simeprevir and in whom lupus-like symptomatology began at an interval of
$3 \mathrm{M} / 24 \mathrm{~W}$ after the termination of antiviral treatment, respectively 2 weeks after starting the therapy, with arthralgia/rash, hematuria, leukocyturia, and progressively aggravated retention of nitrogenous wastes syndrome. It can be assumed that the elimination of viral antigen increases sensitivity to the endogenous effects of IFN in patients with predisposing background, resulting in the unmasking of autoimmunity.

As for the first 2 cases, the patients did not have any known autoimmune diseases, but the second case had an increased level of ANA prior to the diagnosis of glomerulonephritis. In the 3rd patient, DAAs therapy aggravated a previous interferon therapy-induced SLE, with the HCV clearance aggravating a pre-existing autoimmune disease. This is supported by the fact that after a period of 5 years without symptomatology, the patient presented arthralgia and increased retention of nitrogenous wastes syndrome following DAAs therapy. Also, in this case, the renal biopsy did not reveal tubuloreticular inclusions typical of lupus nephritis, and $\mathrm{Clq}$ deposition in the kidneys was minimal. Common changes seen on renal biopsy were: immune complex depositions (mesangial, paramesangial, subendothelial, intramembranous), tubuloreticular inclusions (case 1 and case 2 ) and "full house" pattern typical of lupus nephritis in IF $(\lg \mathrm{G}+, \lg \mathrm{M}+, \lg \mathrm{A}+, \mathrm{C} 3+$, $\mathrm{C} 4+)^{(13)}$ (Table 6, Table 7).

\section{Viral replication and immunosuppre- ssion}

Immunosuppression reduces the inflammatory pathways that lead to liver damage, but on the other hand decreases the immune defence mechanisms against liver injury mediated directly by the virus. Discontinuation of immunosuppressive 


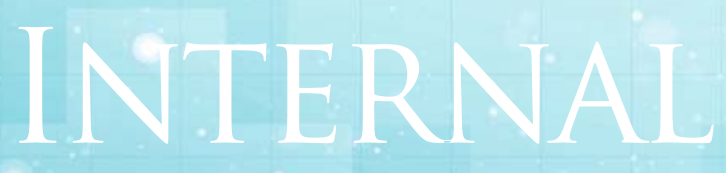

General Reviews

therapy induces a strong rebound immune response that can cause a surge of hepatitis. The incidence of viral reactivation due to immunosuppression is still unknown. There are few studies related to HCV reactivation and which therapeutic interventions are most appropriate for $\mathrm{C}$ virus infection or the underlying disease. There is scant data available in the literature about risk factors, prevalence and clinical outcome of reactivation $^{(14)}$.

Immunosuppression inhibits the immune function of the host and increases the risk of late recurrence of viral infection, which advocates the idea of monitoring the viral load even after reaching SVR. However, viral hepatitis surges do not appear to influence the survival of SLE patients. On the other hand, patients with detectable viremia for long periods of time are at higher risk of mortality due to liver disease, so it is recommended that 2-4 months after starting the immunosuppression, the antiviral treatment to be initiated.

Although viral replication increases in about one-third of patients, cases of severe hepatitis or decompensated liver disease are rare due to a less vigorous immune response to viral antigens (which ultimately leads to a higher rate of chronicization) ${ }^{(5)}$.

The long term use of immunosuppressive agents may damage liver function. Older data from the literature noted an increase in the incidence of cirrhosis and mortality due to liver disease in patients who underwent a kidney transplant, as well as a faster progression to end-stage liver disease. Moreover, the immunosuppressive therapy also has risk of hepatotoxicity (azathioprine, cyclosporin) $)^{(16)}$.

The antiCD20 Ab treatment is associated with a $26 \%$ incidence of hepatitis in patients with $C$ virus infection. It appears that rituximab significantly increases the HCV RNA levels, but even in rare cases of severe hepatitis and decompensated liver disease, patient survival has not been affected.

Cyclosporine inhibits activation of helper $\mathrm{T}$ cells, preventing the recruitment and expansion of cytotoxic $\mathrm{T}$ lymphocytes. During the progressive decrease of the doses or after treatment discontinuation, a massive immune response of the host to viral antigens may occur, leading to massive liver destruction, which advocates the need for antiviral prophylaxis ${ }^{(17)}$ (Table 8).

The ALT level increases during short-term oral corticotherapy, reaching a peak in average 7 weeks after the withdrawal of treatment, indicating that the immune response to the $\mathrm{C}$ virus is slow. Corticosteroids seem to be safe when used in the short term ( $<6$ months), but their discontinuation can cause the rebound of inflammatory processes (Table 9).

DAAs are efficient and safe and increase the probability of viral eradication with few 


\begin{tabular}{|l|l|l|}
\hline $\begin{array}{l}\text { Systemic autoimmune } \\
\text { disease associated with C } \\
\text { virus infection }\end{array}$ & $\begin{array}{l}\text { No. of } \\
\text { patients }\end{array}$ & $\begin{array}{l}\text { Gender } \\
\text { ratio F:M }\end{array}$ \\
\hline Sjogren Syndrome & 77 & $3: 1$ \\
\hline SLE & 43 & $5: 1$ \\
\hline RA & 14 & $5: 2$ \\
\hline APS & 14 & $1: 1$ \\
\hline PAN & 8 & $3: 5$ \\
\hline Sarcoidosis & 7 & $4: 3$ \\
\hline Systemic sclerosis & 7 & $6: 1$ \\
\hline Ankylosing spondylitis & 3 & $1: 2$ \\
\hline Inflammatory myopathy & 2 & $1: 1$ \\
\hline Wegener's granulomatosis & 2 & $2: 0$ \\
\hline Giant cell arteritis & 1 & $0: 1$ \\
\hline Henoch-Schönlein purpura & 1 & $0: 1$ \\
\hline Polymyalgia rheumatica & 1 & $1: 0$ \\
\hline Total & 180 & $3: 1$ \\
\hline & & \\
\hline
\end{tabular}

Table 1. Systemic autoimmune diseases (SAD) in 180 patients with chronic $C$ virus infection ${ }^{(2)}$

Patients diagnosed with SAD and $C$ virus infection, followed up in a multicenter study between 1994 and 2003. SLE systemic lupus erythematosus; RA - rheumatoid arthritis; APS antiphospholipid syndrome; PAN - polyarteritis nodos

Table 2. Clinical, immunological and epidemiological data of SAD-HCV positive patients compared to SAD-HCV negative patients

HCV - hepatitis C virus; CNS central nervous system; ENA extractable nuclear antigen antibodies

\begin{tabular}{|l|l|l|l|}
\hline & $\begin{array}{l}\text { SAD-HCV+ } \\
(\mathbf{n = 1 8 0})\end{array}$ & $\begin{array}{l}\text { SAD-HCV } \\
(\mathbf{n = 1 8 0})\end{array}$ & p value \\
\hline Gender (\% women) & $130(72)$ & $149(83)$ & 0.016 \\
\hline Average age at SAD diagnosis & $50.46+-17.19$ & $46.60+-18.21$ & 0.039 \\
\hline Sicca symptoms & $86(48)$ & $90(50)$ & - \\
\hline Joint damage & $84(47)$ & $97(54)$ & - \\
\hline Raynaud's phenomenon & $23(13)$ & $22(12)$ & - \\
\hline Vasculitis & $38(21)$ & $12(7)$ & $<0.001$ \\
\hline Lung injury & $17(9)$ & $23(13)$ & - \\
\hline Peripheral neuropathy & $17(9)$ & $11(6)$ & - \\
\hline CNS disorders & $9(5)$ & $13(7)$ & - \\
\hline Renal impairment & $23(13)$ & $17(9)$ & - \\
\hline Thrombotic phenomena & $14(8)$ & $11(6)$ & - \\
\hline Skin damage & $44(24)$ & $42(23)$ & - \\
\hline ANA & $116 / 171(68)$ & $134 / 172(78)$ & 0.036 \\
\hline Anti-dsDNA Ab & $24 / 159(15)$ & $43 / 153(32)$ & 0.005 \\
\hline ENA & $35 / 160(22)$ & $50 / 155(32)$ & 0.038 \\
\hline Rheumatoid factor & $90 / 162(56)$ & $57 / 147(39)$ & 0.003 \\
\hline Hypocomplementemia & $87 / 154(57)$ & $46 / 146(31)$ & $<0.001$ \\
\hline Cryoglobulins & $92 / 149(62)$ & $10 / 115(9)$ & $<0.001$ \\
\hline Neoplasia & $27(15)$ & $3(2)$ & $<0.001$ \\
\hline Classification criteria met & $4.02+-1.2$ & $4.61+-1.45$ & $<0.001$ \\
\hline & & & \\
\hline
\end{tabular}




\section{INTERNAL MEDICINE}

General Reviews

\begin{tabular}{|c|c|c|c|}
\hline Manifestation & $\begin{array}{l}\text { SLE patients } \\
(n=36)\end{array}$ & $\begin{array}{l}\text { Patients with SLE and C } \\
\text { virus infection } \\
(n=4)\end{array}$ & $p$ value \\
\hline Neurological $^{b}$ & $6(17)$ & 0 & $>0.99$ \\
\hline Arthritis & $9(25)$ & $4(100)$ & 0.008 \\
\hline Myositis & $3(8)$ & $0(0)$ & $>0.99$ \\
\hline Cutaneous $^{c}$ & $10(28)$ & $0(0)$ & 0.56 \\
\hline Mucosal ulcerations & $5(14)$ & $0(0)$ & $>0.99$ \\
\hline Serositis & $2(6)$ & $1(25)$ & 0.28 \\
\hline Nephropathy ${ }^{d}$ & $17(47)$ & $4(100)$ & 0.11 \\
\hline Cytopenia & $4(11)$ & $0(0)$ & $>0.99$ \\
\hline ANA & $36(100)$ & $4(100)$ & - \\
\hline Anti-dsDNA Ab & $12(33)$ & $1(25)$ & $>0.99$ \\
\hline Anti-SM Ab & $9(25)$ & $0(0)$ & $>0.99$ \\
\hline Low C3 & $23(64)$ & $3(75)$ & $>0.56$ \\
\hline Low C4 & $10(28)$ & $1(25)$ & 0.99 \\
\hline $\begin{array}{l}\text { SLEDAI score in the } \\
\text { time point of testing } \\
\text { for virus } C \text { infection }\end{array}$ & 9.6 & 10.5 & 0.93 \\
\hline
\end{tabular}

Table 3. Clinical, immunological characteristics and SLEDAI score in patients with SLE with/without Ca virus infection(3)

ANA - antinuclear antibodies; SLE - systemic lupus erythematosus; a - the values represent the number (\%) of patients; $b$ - neurological - seizures, psychosis, headache, stroke; c cutaneous - malar rash, discoid lesions, subacute lesions, photosensitivity; $d$ - nephropathy: red blood cell casts, haematuria, proteinuria $>0.5 \mathrm{~g} / 24 \mathrm{~h}$ and/or leucocyturia. 


\begin{tabular}{|c|c|c|c|c|c|c|c|c|c|}
\hline Patient & Gender & Age & SLE criteria & ANA & ENA & $\begin{array}{l}\text { Anti- } \\
\text { dsDNA Ab }\end{array}$ & $\mathrm{aCL}$ & Cryog & Hypocompl \\
\hline 1 & $\mathrm{~F}$ & 62 & Art, Nephr, Hem & 10,000 & $Y$ & $Y$ & $Y$ & $\mathrm{~N}$ & $Y$ \\
\hline 2 & M & 34 & $\begin{array}{l}\text { Art, Nephr, Ser, } \\
\text { Hem }\end{array}$ & 320 & $N$ & $Y$ & $Y$ & III & $Y$ \\
\hline 3 & $\mathrm{~F}$ & 35 & $\begin{array}{l}\text { Art, Ulc, Nephr, } \\
\text { Nrl, Hem }\end{array}$ & 2000 & $Y$ & $N$ & $\mathrm{Y}$ & ॥ & $Y$ \\
\hline 4 & $\mathrm{~F}$ & 65 & Hem & 10,240 & $N$ & $\mathrm{Y}$ & $N$ & III & $\mathrm{N}$ \\
\hline 5 & $\mathrm{~F}$ & 34 & $\begin{array}{l}\text { Art, Nephr, NP, } \\
\text { Ser }\end{array}$ & 1000 & $Y$ & Y & NT & $\mathrm{N}$ & $N$ \\
\hline$\overline{6}$ & $\bar{F}$ & 70 & Art, Nephr, Hem & 100 & $Y$ & $\bar{Y}$ & $\bar{Y}$ & ॥ & $N$ \\
\hline 7 & $\mathrm{~F}$ & 31 & $\begin{array}{l}\text { Art, Photo, } \\
\text { Nephr }\end{array}$ & 5000 & $\mathrm{Y}$ & Y & Y & III & $Y$ \\
\hline 8 & $\mathrm{~F}$ & 36 & $\begin{array}{l}\text { Art, Ulc, Malar, } \\
\text { Hem }\end{array}$ & 2500 & $N$ & $N$ & $N$ & $\mathrm{~N}$ & $Y$ \\
\hline 9 & $\mathrm{~F}$ & 37 & $\begin{array}{l}\text { Art, Subac, } \\
\text { Malar, Hem }\end{array}$ & 160 & $Y$ & $N$ & $Y$ & $\mathrm{~N}$ & $N$ \\
\hline 10 & $M$ & 45 & $\begin{array}{l}\text { Art, Ulc, Malar, } \\
\text { Ser, Hem }\end{array}$ & 320 & $N$ & $\mathrm{Y}$ & $Y$ & $N$ & $N$ \\
\hline 11 & $\mathrm{~F}$ & 53 & $\begin{array}{l}\text { Art, Ser, Ulc, } \\
\text { Subac, NP }\end{array}$ & 1000 & $N$ & Y & $N$ & III & $N$ \\
\hline 12 & $\mathrm{M}$ & 52 & Art, Ser, Hem & 5000 & $\mathrm{Y}$ & $\mathrm{Y}$ & $\mathrm{Y}$ & III & $\mathrm{N}$ \\
\hline 13 & $\mathrm{~F}$ & 33 & Art, Nephr & 640 & $\mathrm{~N}$ & $\mathrm{Y}$ & $Y$ & $\mathrm{~N}$ & $N$ \\
\hline 14 & $\bar{F}$ & 36 & Hem & 1280 & $\mathrm{~N}$ & $\mathrm{~N}$ & $\mathrm{~N}$ & $\overline{\text { II }}$ & $N$ \\
\hline 15 & $\mathrm{~F}$ & 46 & $\begin{array}{l}\text { Art, Subac, } \\
\text { Nephr, Hem }\end{array}$ & 1000 & $Y$ & $\mathrm{Y}$ & $N$ & III & $Y$ \\
\hline 16 & $\mathrm{~F}$ & 20 & $\begin{array}{l}\text { Art, Photo, } \\
\text { Nephr, Hem }\end{array}$ & 1280 & $N$ & $Y$ & $Y$ & $\mathrm{~N}$ & $Y$ \\
\hline 17 & $\mathrm{~F}$ & 46 & Art, Subac & 1280 & $Y$ & $\mathrm{Y}$ & $Y$ & $\mathrm{~N}$ & $N$ \\
\hline 18 & $\bar{F}$ & 25 & Art, Subac & 1280 & $N$ & $Y$ & $\mathrm{Y}$ & III & $\mathrm{Y}$ \\
\hline 19 & $\mathrm{~F}$ & 30 & $\begin{array}{l}\text { Art, NP, Photo, } \\
\text { Hem, Nephr }\end{array}$ & 1280 & $N$ & $\mathrm{Y}$ & $Y$ & $N$ & $N$ \\
\hline
\end{tabular}

Table 4. Clinical manifestations of patients with C virus and SLE(5)

ANA - antinuclear antibodies; ENA - extractable nuclear antigen antibodies; aCL - anticardiolipin antibodies; cryog - cryoglobulin type; hypocompl - hypocomplementemia; art - arthritis; nephrnephropathy; hem - hemocytopenia; Ser - serositis; ulc - mouth ulcers; NP - neuropsychiatric manifestations; Photo - photosensitivity; malar - malar rash; subac - subacute cutaneous lesions; NT- not taken; $Y$ - yes; $N$ - no. Start IFN and RBV 


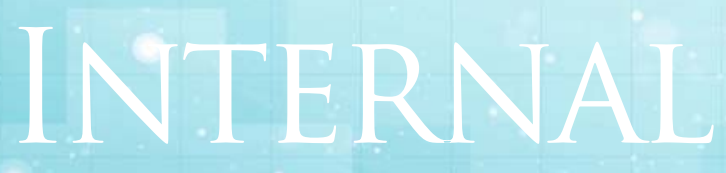

\section{General Reviews}

adverse effects in context of combination with immunosuppressive agents. The study conducted by Colombo et al. reported a SVR (sustained virologic response) in $98 \%$ of patients who underwent a kidney transplant without acute renal rejection ${ }^{(17)}$ (Table 10). The DAAs' success in achieving SVR demonstrates that inhibition of viral replication without further inducing an immune response is enough to eradicate HCV. However, it is still unclear how intact the immune system should be for an effective viral eradication using DAAs.

SLE is a disease requiring immunosuppressive therapy, but it is still unclear whether SLE patients are at higher risk of HCV reactivation or if the immunological profile influences the reactivation.

The study conducted by Chen et al. followed 26 patients with $C$ virus and SLE over an average time of 8.4 years, of whom 10 met the criteria of viral infection reactivation and 16 patients did not meet these criteria (reactivation of viral infection - recurrence of HCV RNA if it was previously undetectable or an elevation of $>1 \log 10 \mathrm{IU} / \mathrm{mL}$ compared to baseline) and 16 did not meet these criteria. It has been noted that the immunosuppression period was similar in both groups (5.2 years vs. 5.4 years) and that there was no statistically significant difference regarding age, gender, liver function, SLE immunological profile. Moreover, lupus activity was exacerbated by hepatitis surges and neither by interferon and ribavirin therapy. Of the total patients, $20 \%$ of those with genotype $1 \mathrm{~b}$ and $66.7 \%$ of those with genotype 2 a obtained RVR (rapid virologic response - undetectable HCV RNA in the 4th week of antiviral treatment), similar to the general population with $\mathrm{C}$ virus.

However, none of the patients treated with glucocorticoids reached RVR, probably because glucocorticoids attenuate the host immune function and increase viral replication, leading to a poor initial response to antiviral therapy, which increases the risk of late relapse (recurrence of HCV RNA after obtaining SVR - 24 W after antiviral therapy) $)^{(18)}$ (Table 11).

\section{Long term prognosis}

Patients with $\mathrm{C}$ virus present a faster progression to chronic end-stage kidney disease, with 10 year renal survival being statistically significantly lower, which is consistent with the study conducted by Tsui et al., demonstrating that $C$ virus infection is associated with a 3 times higher risk of progression to chronic end-stage kidney disease. $C$ virus infection is also associated with a higher incidence of cardiovascular diseases (increased risk of atherosclerosis) and diabetes (insulin resistance - it reduces the insulin receptor expression) ${ }^{(19)}$. 


\begin{tabular}{|c|c|c|c|c|c|c|c|c|c|c|c|c|}
\hline Month & $\begin{array}{l}\mathrm{Hgb} \\
(\mathrm{g} / \mathrm{L})\end{array}$ & $\begin{array}{l}\text { WBC } \\
\left(10^{3} / \mu L\right)\end{array}$ & $\begin{array}{l}\text { PLT } \\
\left(\times 10^{3} / \mu L\right)\end{array}$ & $\begin{array}{l}\text { ALT } \\
\text { (U/L) }\end{array}$ & $\begin{array}{l}\text { GGT } \\
\text { (U/L) }\end{array}$ & $\begin{array}{l}\text { Serum } \\
\text { creatinine } \\
\text { (mmol/L) }\end{array}$ & $\begin{array}{l}\text { Serum } \\
\text { total } \\
\text { protein } \\
(\mathrm{g} / \mathrm{L}) \\
\end{array}$ & $\begin{array}{l}\text { Serum } \\
\text { albu- } \\
\text { min } \\
\text { (g/L) } \\
\end{array}$ & $\begin{array}{l}\text { Protej- } \\
\text { nuria } \\
\text { (g/24h) }\end{array}$ & $\begin{array}{l}\text { RNA HCV } \\
\text { (copies/mL) }\end{array}$ & ANA & $\begin{array}{l}\text { Anti- } \\
\text { dsDNA } \\
\mathrm{Ab} \\
\text { (IU/mL) } \\
\end{array}$ \\
\hline 0 & 10.2 & 2.4 & 93 & 10 & 15 & 129.6 & 46 & 14 & 7.5 & Positive & 1L1280 & 774.82 \\
\hline 5 & 12.9 & 4.8 & 208 & 36 & 61 & 83.9 & 48 & 24 & 5.1 & - & $1: 640$ & Negative \\
\hline 12 & 14.6 & 6.6 & 239 & 39 & 53 & 91.5 & 49 & - & 4.6 & $1.02 \times 10^{4}$ & $1: 320$ & Negative \\
\hline 17 & 14.5 & 4.8 & 189 & 37 & 38 & 100.7 & 49 & 26 & 4.5 & $1.12 \times 10^{6}$ & $1: 320$ & 104.67 \\
\hline 21 & 14.3 & 6.76 & 232 & 44 & 78 & 88.5 & 55 & 30 & 4.0 & $2.21 \times 10^{6}$ & $1: 320$ & 162.83 \\
\hline 29 & 10.4 & 4.2 & 162 & 19 & 44 & 72.4 & 51 & 27 & $\begin{array}{l}\text { No } \\
\text { data }\end{array}$ & $\begin{array}{l}\text { Not } \\
\text { detectable }\end{array}$ & $1: 100$ & 52.14 \\
\hline 37 & 12.9 & 2.3 & 140 & 22 & 75 & 70.9 & 59 & 36 & 2.0 & $\begin{array}{l}\text { Not } \\
\text { detectable }\end{array}$ & $1: 320$ & 80.16 \\
\hline 54 & 13.7 & 4.77 & 296 & 34 & 97 & 70.9 & 60 & 35 & Absent & $\begin{array}{l}\text { Not } \\
\text { detectable }\end{array}$ & $1: 100$ & 63.77 \\
\hline Normal & $\begin{array}{l}12- \\
16\end{array}$ & $4-10$ & $\begin{array}{l}140- \\
440\end{array}$ & $\begin{array}{l}0- \\
35\end{array}$ & $\begin{array}{l}0- \\
38\end{array}$ & $62-99$ & $66-83$ & $35-52$ & Absent & $\begin{array}{l}\text { Not } \\
\text { detectable }\end{array}$ & negative & $<100$ \\
\hline
\end{tabular}

Table 5. Laboratory test results after initiation of antiviral treatment ${ }^{(6)}$

Hgb - haemoglobin; WBC - white blood cell; PLT - platelets; IFN - interferon; RBV - ribavirin.

\begin{tabular}{|c|c|c|}
\hline $\begin{array}{l}\text { Case } \\
\text { no. }\end{array}$ & Serological baseline values & Serological changes after DAAs administration \\
\hline \multirow[t]{2}{*}{1} & $\begin{array}{l}\text { Cryoglobulins: negative } \\
\text { Other serology: unavailable }\end{array}$ & $\begin{array}{l}\text { ANA: 1:320 Anti-DNA Ab : negative Anti-histone Ab : NT } \\
\text { Anti-SM Ab: negative Complement C } 3: 74 \text { (low), C4: } 14 \\
\text { Cryoglobulins: negative RF: negative Anti-SM Ab :1:80 } \\
\text { ANCA: negative }\end{array}$ \\
\hline & $\begin{array}{l}\text { ANA: 1:640 in } 2007 \text { Cryoglobulins: negative } \\
\text { RF: positive in } 2007 \\
\text { Other serology: unavailable }\end{array}$ & $\begin{array}{l}\text { ANA: > 1:5120 Positive Anti-DNA Ab :1:80 Anti-histone } \\
\text { Ab: }>7.0 \\
\text { Anti Ro/La: negative Anti-SM: negative C3: } 54 \text { C4: } 9 \text { (low) } \\
\text { Cryoglobulins: negative } \\
\text { RF: negative }\end{array}$ \\
\hline 3 & $\begin{array}{l}\text { ANA: 1:160. Anti -dsDNA Ab : negative } \\
\text { Anti Ro/La Ab: negative Anti-SM Ab: negative } \\
\text { Cryoglobulins: } 5 \% \text { RF: negative } \\
\text { Complement: C3: } 87 \mathrm{C} 4: 20 \text { (normal) } \\
\text { Other serology: unavailable }\end{array}$ & $\begin{array}{l}\text { ANA: 1:160 Anti-dsDNA Ab: negative Anti-histone Ab: } \\
\text { 1.5U } \\
\text { Anti Ro/La: positive Anti-SM Ab: negative Cryoglobulins: } \\
1 \% \\
\text { C3: } 42 \text { (low) C4: } 7 \text { (low) } \\
\text { RF: negative }\end{array}$ \\
\hline
\end{tabular}

Table 6. Comparison of serological changes before and after DAAs treatment ${ }^{(13)}$

Ab - antibody; NT - not taken; complement normal values: c3 81-157 mg/dL; c4=12-39 $\mathrm{mg} / \mathrm{dL}$; Anti-histone $\mathrm{Ab} \mathrm{N}<1.0 \cup$ (Presence of Antihistone Ab has raised the likelihood of drug-induced lupus, but this is rarely associated with glomerulonephritis) 
General Reviews

\begin{tabular}{|c|c|c|c|c|}
\hline Case & Patient characteristics & $\begin{array}{l}\text { Clinical } \\
\text { presentation }\end{array}$ & Immunosuppression & Outcome \\
\hline \multirow[t]{4}{*}{1} & Asian female, 51 years old & Morbilliform rash & $\begin{array}{l}\text { MP } 500 \mathrm{mg} \text { iv } \times 3 \text { days, } \\
\text { followed by prednisone } 60 \\
\text { mg/day }\end{array}$ & $\begin{array}{l}\text { Rapid improvement of } \\
\text { kidney function }\end{array}$ \\
\hline & $\begin{array}{l}\text { Genotype 3a, viral load } 3.6 \\
\times 10^{5}\end{array}$ & $\begin{array}{l}\text { Elevated } \\
\text { transaminases }\end{array}$ & & SVR12 \\
\hline & $\begin{array}{l}\text { Previous treatment: Peg- } \\
\text { IFN/RBV }\end{array}$ & $\begin{array}{l}\text { Increasing } \\
\text { creatinine }\end{array}$ & & $\begin{array}{l}\text { Death due to sepsis, fungal } \\
\text { pneumonia and empyema }\end{array}$ \\
\hline & $\begin{array}{l}\text { Cirrhosis diagnosed on } \\
\text { biopsy }\end{array}$ & $\begin{array}{l}\text { Proteinuria and } \\
\text { hematuria }\end{array}$ & & \\
\hline \multirow[t]{4}{*}{2} & $\begin{array}{l}\text { Caucasian female, } 56 \\
\text { years old }\end{array}$ & Joint pain & $\begin{array}{l}\text { MP } 250 \mathrm{mg} \text { iv } \times 3 \text { days, } \\
\text { followed by prednisone } 60 \\
\mathrm{mg} / \text { day }\end{array}$ & $\begin{array}{l}\text { Rapid improvement of } \\
\text { kidney function }\end{array}$ \\
\hline & $\begin{array}{l}\text { Genotype } 1 \mathrm{~b} \text {, viral load } 2.8 \\
\times 10^{6}\end{array}$ & $\begin{array}{l}\text { Increasing } \\
\text { creatinine }\end{array}$ & $\begin{array}{l}\text { Prednisone was decreased } \\
\text { rapidly to } 20 \mathrm{mg} / \text { day due to } \\
\text { the occurrence of delirium, } \\
\text { then discontinued until W4 }\end{array}$ & $\begin{array}{l}\text { HCV relapse, treated with } \\
\text { Sofosbuvir and Ledipasvir }\end{array}$ \\
\hline & $\begin{array}{l}\text { Previous treatment: Peg- } \\
\text { IFN/RBV }\end{array}$ & $\begin{array}{l}\text { Hematuria and } \\
\text { leucocyturia }\end{array}$ & $\begin{array}{l}\text { MMF maintenance therapy, } \\
250 \mathrm{mg} \times 2 / \text { day, } 5 \text { months }\end{array}$ & $\begin{array}{l}\text { Recurrence of joint pain } \\
\text { under DAAs; resuming } \\
\text { steroids and MMF with } \\
\text { improving symptoms }\end{array}$ \\
\hline & $\begin{array}{l}\text { Cirrhosis diagnosed on } \\
\text { allograft biopsy }\end{array}$ & & & SVR12 \\
\hline \multirow[t]{4}{*}{3} & $\begin{array}{l}\text { Hispanic female, } 47 \text { years } \\
\text { old }\end{array}$ & $\begin{array}{l}\text { Rash (face, chest, } \\
\text { hands) }\end{array}$ & $\begin{array}{l}\text { MP } 250 \mathrm{mg} \text { iv } \times 3 / \text { day, followed } \\
\text { by prednisone } 60 \mathrm{mg} / \text { day }\end{array}$ & $\begin{array}{l}\text { Improvement of kidney } \\
\text { function }\end{array}$ \\
\hline & $\begin{array}{l}\text { Genotype } 1 \text {, viral load } 8.4 \\
\times 10^{4}\end{array}$ & $\begin{array}{l}\text { Low back pain, } \\
\text { physical fatigue }\end{array}$ & $\begin{array}{l}\text { Rituximab } 1000 \mathrm{mg} \text { iv/week x } \\
3\end{array}$ & SVR 12 \\
\hline & Previous treatment: IFN $\alpha$ & $\begin{array}{l}\text { Increasing } \\
\text { creatinine }\end{array}$ & & $\begin{array}{l}\text { Death due to bacteraemia, } \\
\text { bacterial pneumonia }\end{array}$ \\
\hline & $\begin{array}{l}\text { Clinically diagnosed } \\
\text { cirrhosis }\end{array}$ & $\begin{array}{l}\text { Proteinuria and } \\
\text { hematuria }\end{array}$ & & \\
\hline
\end{tabular}

Table 7. Patient outcome with lupus-like syndrome after administration of DAAs

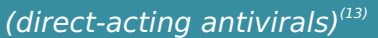

MP - methylprednisolone; MMF - mycophenolat mofetil; IFN - interferon; HCV hepatitis C virus; SVR - sustained virologic response 


\begin{tabular}{|c|c|c|c|c|c|c|c|c|c|c|}
\hline Study & Туре & $\mathbf{N}$ & Age & $\begin{array}{l}\text { Males } \\
\%\end{array}$ & $\begin{array}{l}\text { Immunosupp } \\
\text { ression }\end{array}$ & $\begin{array}{l}\text { AT (antiviral } \\
\text { therapy) }\end{array}$ & Outcome & $\begin{array}{l}\text { Adverse } \\
\text { effects }\end{array}$ & $\begin{array}{l}\text { SVR } \\
\%\end{array}$ & $\begin{array}{l}\text { Clinical } \\
\text { response }\end{array}$ \\
\hline $\begin{array}{l}\text { Sise, } \\
2016\end{array}$ & $\begin{array}{l}\text { Case } \\
\text { series }\end{array}$ & 5 & N/A & N/A & $\begin{array}{l}\text { Rituximab } x \\
4 \text { patients } \\
\text { Rituximab } x \\
1 \text { patient }\end{array}$ & $\begin{array}{l}\text { Sofosbuvir } \\
+ \text { RBV; } \\
\text { Or } \\
\text { Sofosbuvir+ } \\
\text { Simeprevir x } \\
12 \mathrm{~W}\end{array}$ & $\begin{array}{l}\text { SVR12 in } 3 / 4 \\
\text { patients on } \\
\text { Rituximab } \\
\text { and } 0 / 1 \\
\text { patients on } \\
\text { Ustekinumab } \\
\text { (reactivation) }\end{array}$ & $\begin{array}{l}\text { Not } \\
\text { reported }\end{array}$ & $60 \%$ & - \\
\hline $\begin{array}{l}\text { Saadoun, } \\
2014\end{array}$ & $\begin{array}{l}\text { Cohort, } \\
\text { pros- } \\
\text { pective } \\
\text { study }\end{array}$ & 4 & N/A & $\mathrm{N} / \mathrm{A}$ & $\begin{array}{l}\text { Rituximab } \\
\text { adminis- } \\
\text { tered one } \\
\text { month } \\
\text { before AT }\end{array}$ & $\begin{array}{l}\text { Peg } \\
\text { IFN } \alpha+\text { RBV } x \\
48 W+\text { Tela- } \\
\text { previr x } 12 \mathrm{~W} \\
\text { or Bocepre- } \\
\text { vir } 44 \mathrm{~W}\end{array}$ & $\begin{array}{l}\text { PR in } 2 / 4 \\
C R \text { in } 2 / 4\end{array}$ & $\begin{array}{l}\text { Not } \\
\text { reported }\end{array}$ & - & $100 \%$ \\
\hline $\begin{array}{l}\text { Humphrie } \\
2014\end{array}$ & $\begin{array}{l}\text { Case } \\
\text { report }\end{array}$ & 1 & 59 & $100 \%$ & $\begin{array}{l}\text { MP } 500 \text { mg } x \\
2 \text { days and } 5 \\
\text { days plas- } \\
\text { mapheresis }\end{array}$ & $\begin{array}{l}\text { Telaprevir, } \\
\text { IFN, RBV x } \\
24 W\end{array}$ & $\begin{array}{l}\text { PR. } \\
\text { Progression } \\
\text { to st. } 5 \text { CKD } \\
\text { and HD at W7 } \\
\text { after the end } \\
\text { of the } \\
\text { treatment. } \\
\text { SVR. }\end{array}$ & $\begin{array}{l}\text { Anemia } \\
\text { with } \\
\text { transfusion } \\
\text { require- } \\
\text { ments, } \\
\text { EPO, inter- } \\
\text { ruption of } \\
\text { IFN and } \\
\text { RBV }\end{array}$ & $100 \%$ & $0 \%$ \\
\hline $\begin{array}{l}\text { Zaidan, } \\
2012\end{array}$ & $\begin{array}{l}\text { Case } \\
\text { report }\end{array}$ & 2 & 44 & $50 \%$ & $\begin{array}{l}\text { Cyclophosph } \\
\text { amide and } \\
\text { pulse } \\
\text { steroid, } \\
\text { afterwards } \\
\text { prednisone } \\
50 \text { mg/day; } \\
\text { case 4: } \\
\text { prednisone } 1 \\
\text { mg/kg } \\
\text { bw/day x } 5 \\
\text { days, plas- } \\
\text { mapheresis, } \\
\text { rituximab iv } \\
\end{array}$ & $\begin{array}{l}\text { Peg IFN } \alpha \\
+R B\end{array}$ & $\begin{array}{l}\text { Case 3: SVR. } \\
\text { Purpura } \\
\text { remitted. } \\
\text { Kidney } \\
\text { function PR. } \\
\text { Case 4: } \\
\text { Cutaneous } \\
\text { lesions } \\
\text { remitted. } \\
\text { Recovered } \\
\text { kidney } \\
\text { function. }\end{array}$ & $\begin{array}{l}\text { Not } \\
\text { reported }\end{array}$ & - & $100 \%$ \\
\hline $\begin{array}{l}\text { Rodrigo, } \\
2012\end{array}$ & $\begin{array}{l}\text { Case } \\
\text { report }\end{array}$ & 1 & 34 & $100 \%$ & $\begin{array}{l}\text { Prednisone } \\
10 \mathrm{mg} \mathrm{x} \\
2 / \text { day }+ \\
\text { Cyclophosph } \\
\text { amide }\end{array}$ & $\begin{array}{l}\text { Peg IFN } \\
2 \alpha+\text { RBV }\end{array}$ & $\begin{array}{l}\text { Healed } \\
\text { cutaneous } \\
\text { lesions. } \\
\text { Improved } \\
\text { muscle } \\
\text { weakness. No } \\
\text { relapse at } 6 \\
\text { months. }\end{array}$ & $\begin{array}{l}\text { Not } \\
\text { reported }\end{array}$ & - & $100 \%$ \\
\hline $\begin{array}{l}\text { Kiremitci, } \\
2012\end{array}$ & $\begin{array}{l}\text { Case } \\
\text { report }\end{array}$ & 1 & 34 & $0 \%$ & $\begin{array}{l}\text { MP } 0.5 \mathrm{mg} / \mathrm{kg} \\
\text { bw/day } \\
\text { starting with } \\
\text { the AT }\end{array}$ & PegIFN $2 \alpha$ & $\begin{array}{l}\text { Remitted } \\
\text { nodules. } \\
\text { Normalised } \\
\text { kidney } \\
\text { function, but } \\
\text { viral } \\
\text { reactivation }\end{array}$ & $\begin{array}{l}\text { Not } \\
\text { reported }\end{array}$ & - & $0 \%$ \\
\hline
\end{tabular}

Table 8. Cryoglobulinemia and vasculitis - immunosuppression and antiviral therapy ${ }^{(17)}$

MP - methylprednisolone; PR - partial remission; CR - complete remission; IFN - interferon; RBV - ribavirin; CKD - chronic kidney disease; HD - hemodialysis; EPO - erythropoietin; SVR sustained virologic response 


\begin{tabular}{|c|c|c|c|c|c|c|c|c|c|c|}
\hline Study & Type & $\mathbf{N}$ & Age & $\begin{array}{l}\text { Males } \\
\%\end{array}$ & Disease & $\begin{array}{l}\text { Immuno } \\
\text { suppression }\end{array}$ & $\begin{array}{l}\text { AT (antiviral } \\
\text { therapy) }\end{array}$ & $\begin{array}{l}\text { Out - } \\
\text { come }\end{array}$ & $\begin{array}{l}\text { Adverse } \\
\text { effects }\end{array}$ & $\begin{array}{l}\text { SVR } \\
\%\end{array}$ \\
\hline $\begin{array}{l}\text { Basu, } \\
2016\end{array}$ & $\begin{array}{l}\text { Rando } \\
\text { mized } \\
\text { control } \\
\text { led } \\
\text { trial }\end{array}$ & $\begin{array}{l}3 \\
5\end{array}$ & 50 & $74 \%$ & $\begin{array}{l}\text { Infla- } \\
\text { mmato- } \\
\text { ry bowel } \\
\text { disease }\end{array}$ & $\begin{array}{l}\text { Antagonist } \\
\text { TNF alpha } \\
\text { Maintained } \\
\text { during AT }\end{array}$ & $\begin{array}{l}\text { LDV+SOV+ } \\
\text { RBV } 1000 \mathrm{mg} \\
\times 8 W \text { or } \\
\text { LDV+SOF } \\
12 W\end{array}$ & $\begin{array}{l}\text { RVS12 in } \\
34 / 35\end{array}$ & $\begin{array}{l}\mathrm{Hb}<8.5 \text { in } 2 \\
\text { pt; } \\
\text { The severity } \\
\text { of other } \\
\text { adverse } \\
\text { effects is } \\
\text { unclear }\end{array}$ & $97 \%$ \\
\hline $\begin{array}{l}\text { Sise, } \\
2016\end{array}$ & $\begin{array}{l}\text { Case } \\
\text { series }\end{array}$ & 5 & N/A & $N / A$ & $\begin{array}{l}\text { Cryo- } \\
\text { globuli- } \\
\text { nemia } \\
\text { Mixt }\end{array}$ & $\begin{array}{l}\text { Rituximab x } \\
4 \text { pt } \\
\text { Ustekinumab } \\
\text { x } 1 \text { pt }\end{array}$ & $\begin{array}{l}\text { Sofosbuvir+ } \\
\text { RBV or } \\
\text { Sofosbuvir+ } \\
\text { simeprevir x } \\
12 \text { W }\end{array}$ & $\begin{array}{l}\text { RVS12 in } \\
3 / 4 \text { pt on } \\
\text { Rituxi- } \\
\text { mab and } \\
0 / 1 \text { pt on } \\
\text { Usteki- } \\
\text { numab } \\
\text { (reacti- } \\
\text { vation) }\end{array}$ & $\begin{array}{l}\text { Not reported } \\
\text { individually }\end{array}$ & $60 \%$ \\
\hline $\begin{array}{l}\text { Hahn, } \\
2015\end{array}$ & $\begin{array}{l}\text { Case } \\
\text { series }\end{array}$ & 1 & 50 & $100 \%$ & RA & $\begin{array}{l}\text { HQ, MP } 8 \mathrm{mg} \text { x } \\
4 / \text { day, } \\
\text { Sulfasalazine }\end{array}$ & $\begin{array}{l}\text { Sofosbuvir/ } \\
\text { ledipasvir/GS- } \\
9451 \times 6 W\end{array}$ & SVR24 & $\begin{array}{l}\text { Surge of } \\
\text { arthritis at } \\
1 \mathrm{~W} \text { after } \\
\text { finishing AT }\end{array}$ & $\begin{array}{l}100 \\
\%\end{array}$ \\
\hline
\end{tabular}

Table 9. Immunosuppression and DAAs ${ }^{(17)}$

RA - rheumatoid arthritis; HQ - hydroxychloroquine; MP - methylprednisolone; LDV -

ledipasvir; SOVISOF - sofosbuvir; RBV - ribavirin; Hb - hemoglobin; pt - patients; W - week 


\begin{tabular}{|c|c|c|c|c|c|c|c|c|c|c|c|}
\hline Study & Type & $\mathbf{N}$ & Age & $\begin{array}{l}\text { Males } \\
(\%)\end{array}$ & IS reason & $\begin{array}{l}\text { Immuno- } \\
\text { suppression } \\
\text { (IS) }\end{array}$ & $\begin{array}{l}\text { AT (antiviral } \\
\text { therapy) }\end{array}$ & Outcome & $\begin{array}{l}\text { Adverse } \\
\text { reactions }\end{array}$ & SVR & $\begin{array}{l}\text { Clinical } \\
\text { res- } \\
\text { ponse }\end{array}$ \\
\hline $\begin{array}{l}\text { Wu, } \\
2013\end{array}$ & $\begin{array}{l}\text { Ca- } \\
\text { se } \\
\text { re- } \\
\text { port }\end{array}$ & 1 & 57 & $0 \%$ & $\begin{array}{l}\text { MPGN } \\
\text { cryo- } \\
\text { globuli- } \\
\text { nemic }\end{array}$ & $\begin{array}{l}\text { MP } \\
40 \mathrm{mg} / \mathrm{day}\end{array}$ & $\begin{array}{l}\text { Peg IFN } \alpha \\
2 b+R B V\end{array}$ & $\begin{array}{l}\text { Remitted } \\
\text { thrombo- } \\
\text { cytopenia, } \\
\text { improve-- } \\
\text { ment of } \\
\text { kidney } \\
\text { function. } \\
\text { Persisten- } \\
\text { tly eleva- } \\
\text { ted cryo- } \\
\text { globulins } \\
\end{array}$ & $\begin{array}{l}\text { Not } \\
\text { reported }\end{array}$ & - & $100 \%$ \\
\hline $\begin{array}{l}\text { Kiremitci } \\
2012\end{array}$ & $\begin{array}{l}\text { Ca- } \\
\text { se } \\
\text { re- } \\
\text { port }\end{array}$ & 1 & 34 & $0 \%$ & $\begin{array}{l}\text { MPGN } \\
\text { cryo- } \\
\text { globuli- } \\
\text { nemic, } \\
\text { purpu- } \\
\text { ra, } \\
\text { pneu- } \\
\text { monitis }\end{array}$ & $\begin{array}{l}\text { MP } \\
0.5 \mathrm{mg} / \mathrm{kg} / \\
\text { day }\end{array}$ & PegIFN-2 $\alpha$ & $\begin{array}{l}\text { Remitted } \\
\text { nodules. } \\
\text { Normalised } \\
\text { kidney } \\
\text { function, } \\
\text { afterwards } \\
\text { relapse }\end{array}$ & $\begin{array}{l}\text { Not } \\
\text { reported }\end{array}$ & - & $0 \%$ \\
\hline $\begin{array}{l}\text { Ahmed } \\
2008\end{array}$ & $\begin{array}{l}\text { Ca- } \\
\text { se } \\
\text { re- } \\
\text { port }\end{array}$ & 1 & 35 & $100 \%$ & $\begin{array}{l}\text { MPGN } \\
\text { type } 1 \\
\text { nega- } \\
\text { tive } \\
\text { cryo- } \\
\text { globulin }\end{array}$ & $\begin{array}{l}\text { MP before } \\
\text { AT } \\
\text { Prednisone } \\
40 \mathrm{mg} / \mathrm{day} \\
\text { starting } \\
\text { with AT }\end{array}$ & $\begin{array}{l}\text { PegIFN } \alpha \text {, } \\
\text { RBV added } \\
\text { after } 3 \\
\text { months }\end{array}$ & $\begin{array}{l}\text { Improved } \\
\text { kidney } \\
\text { function. } \\
\text { SVR. } \\
\text { Elevated } \\
\text { albumin } \\
\text { (38g/L) }\end{array}$ & $\begin{array}{l}\text { No major } \\
\text { adverse } \\
\text { effect }\end{array}$ & $\begin{array}{l}100 \\
\%\end{array}$ & $100 \%$ \\
\hline $\begin{array}{l}\text { Oeda, } \\
2012\end{array}$ & $\begin{array}{l}\text { Ca- } \\
\text { se } \\
\text { re- } \\
\text { port }\end{array}$ & 2 & 50 & $0 \%$ & $\begin{array}{l}\text { Auto- } \\
\text { immune } \\
\text { hepa- } \\
\text { titis } \\
+ \text { HCV }\end{array}$ & $\begin{array}{l}\text { Predniso- } \\
\text { lone } 30-40 \\
\mathrm{mg} / \mathrm{day} \\
\text { before AT } \\
\text { and } \\
\text { reduced to } \\
20 \mathrm{mg} / \mathrm{day} \\
\text { when } \\
\text { starting AT } \\
\end{array}$ & $\begin{array}{l}\text { Peg IFN } \alpha \\
2 b+R B V x \\
24-48 W\end{array}$ & $\begin{array}{l}\text { SVR in } \\
\text { both cases. } \\
\text { Normalised } \\
\text { ALT and Ig } \\
\text { G. }\end{array}$ & $\begin{array}{l}\text { Not } \\
\text { reported }\end{array}$ & $\begin{array}{l}100 \\
\%\end{array}$ & $100 \%$ \\
\hline $\begin{array}{l}\text { Scherze } \\
2008\end{array}$ & $\begin{array}{l}\text { Ca- } \\
\text { se } \\
\text { re- } \\
\text { port }\end{array}$ & 3 & 47 & $67 \%$ & $\begin{array}{l}\text { Crohn's } \\
\text { disease }\end{array}$ & $\begin{array}{l}\text { Azathio- } \\
\text { prine }\end{array}$ & $\begin{array}{l}\text { PEG-IFN } \\
\alpha 2 a+R B V \\
\times 24 W\end{array}$ & $\begin{array}{l}\text { SVR, NR, } \\
\text { relapse }\end{array}$ & $\begin{array}{l}\text { Surge of } \\
\text { Crohn's } \\
\text { disease in } \\
2 / 3 \text { of } \\
\text { patients }\end{array}$ & $33 \%$ & - \\
\hline $\begin{array}{l}\text { Azhar, } \\
2010\end{array}$ & $\begin{array}{l}\text { Ca- } \\
\text { se } \\
\text { re- } \\
\text { port }\end{array}$ & 1 & 40 & $0 \%$ & $\begin{array}{l}\text { Auto- } \\
\text { immune } \\
\text { hepa- } \\
\text { titis } \\
+\mathrm{HCV}\end{array}$ & MMF & IFN+RBV & $\begin{array}{l}\text { SVR. No } \\
\text { surge of } \\
\text { hepatitis. }\end{array}$ & $\begin{array}{l}\text { Cytopenia } \\
\text { requiring } \\
\text { Filgrastim } \\
\text { and } \\
\text { erythropo- } \\
\text { ietin }\end{array}$ & $\begin{array}{l}100 \\
\%\end{array}$ & - \\
\hline $\begin{array}{l}\text { Scherze, } \\
2008\end{array}$ & $\begin{array}{l}\text { Ca- } \\
\text { se } \\
\text { se- } \\
\text { ries }\end{array}$ & 1 & 56 & $100 \%$ & $\begin{array}{l}\text { Crohn's } \\
\text { disease }\end{array}$ & MMF & $\begin{array}{l}\text { PEG- } \\
\text { IFNa2a+ } \\
\text { RBV } 48 \mathrm{~W}\end{array}$ & SVR & $\begin{array}{l}\text { Surge of } \\
\text { Crohn's } \\
\text { disease - } \\
\text { stenosis }\end{array}$ & $\begin{array}{l}100 \\
\%\end{array}$ & - \\
\hline
\end{tabular}

Table 10. Immunosuppression and antiviral treatment in C virus infection ${ }^{(17)}$

MP - methylprednisolone; MPGN - membranoproliferative glomerulonephritis; IFN - interferon; RBV - ribavirin; SVR - sustained virologic response; MMF - mycophenolat mofetil; NR - no response; HCV - hepatitis C virus 


\section{INTERNAL ME}

General Reviews

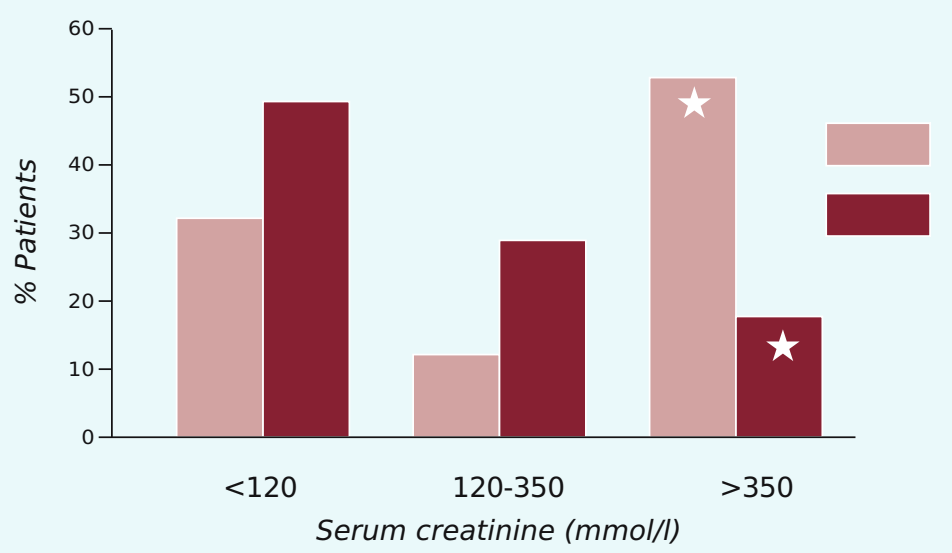

Hep C+

Figure 1. Kidney function in patients with lupus nephritis with and without virus $C$ infection at the last follow$u p^{(21)}$ Statistically significant difference between the group with $C$ virus infection and the one without $C$ virus infection in patients with serum creatinine $>350 \mathrm{mmol} / \mathrm{L}$ $(p<0.02)$

Figure 2. Creatinine

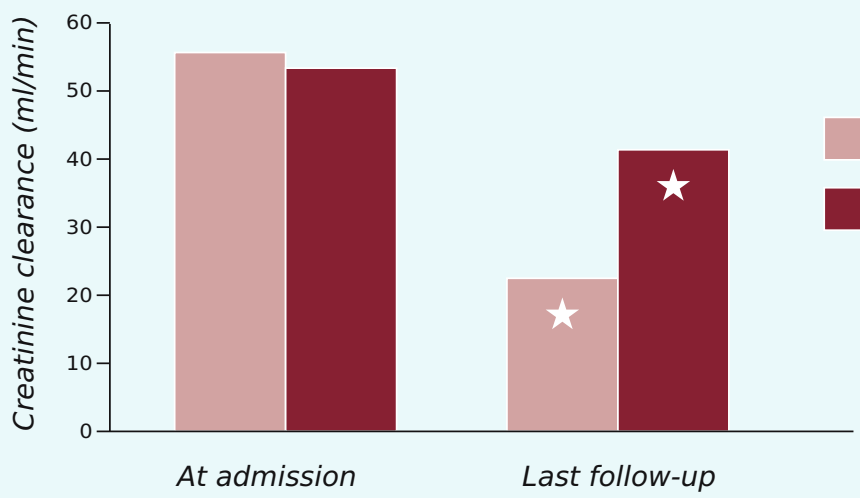

clearance in patients with lupus nephritis with/without Hep C+ virus C infection at the enrolment in the study and at the end of the study Statistically significant difference between the patients with $C$ virus and those without $C$ virus at the last follow-up $(p<0.043)$.

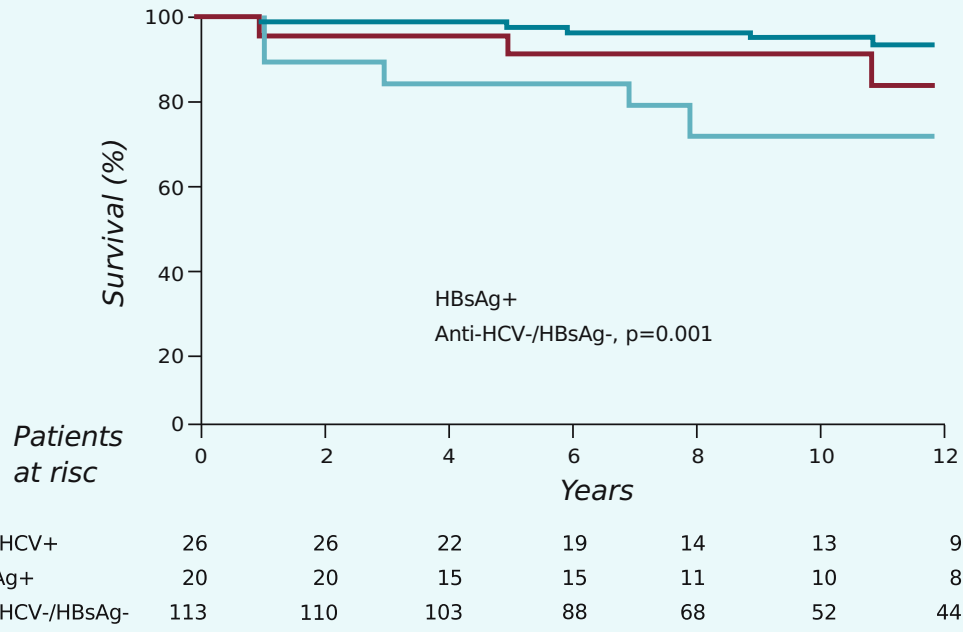

Figure 3. Long term survival for patients with $S L E^{(18)}$ Patients with positive Hbs Ag had lower survival compared to patients negative for $H B$ and also negative for Anti HCV $A b$

$\begin{array}{lrrrrrrr}\text { Anti-HCV }+ & 26 & 26 & 22 & 19 & 14 & 13 & 9 \\ \text { HBsAg+ } & 20 & 20 & 15 & 15 & 11 & 10 & 8 \\ \text { Anti-HCV-/HBsAg- } & 113 & 110 & 103 & 88 & 68 & 52 & 44\end{array}$




\begin{tabular}{|c|c|c|c|}
\hline & $\begin{array}{l}\text { HCV activation } \\
\text { Yes }(n=10)\end{array}$ & No $(n=16)$ & $p$ value \\
\hline Age at diagnosis & $42.5+-17.1$ & $35.0+-13.2$ & 0.201 \\
\hline Females $(n)$ & $8(80 \%)$ & $15(93.8 \%)$ & 0.538 \\
\hline ALT (U/L) & $40.6+-23.1$ & $29.4+-13.4$ & 0.436 \\
\hline AST (U/L) & $37.6+-12.6$ & $27.8+-10.2$ & 0.156 \\
\hline ANA $>1: 160$ & $6 / 10(60 \%)$ & $15 / 16(93.8 \%)$ & 0.055 \\
\hline Anti SSA/Ro positive & $6 / 9(66.7 \%)$ & $6 / 9(66.7 \%)$ & 1.000 \\
\hline Anti SSB/La positive & $2 / 8(25 \%)$ & $1 / 6(16.7 \%)$ & 1.000 \\
\hline Anti-SM positive & $1 / 9(11.1 \%)$ & $2 / 8(25 \%)$ & 0.576 \\
\hline Anti-RNP positive & 1/9 (11.1\%) & $3 / 8(37.5 \%)$ & 0.294 \\
\hline Anti dsDNA positive & 9/10 (90\%) & $10 / 16(62.5 \%)$ & 0.190 \\
\hline Treatment before IS & $7(70 \%)$ & $11(68.8 \%)$ & 1.000 \\
\hline $\begin{array}{l}\text { Duration of IS } \\
\text { treatment }\end{array}$ & $5.4+-6.8$ & $5.2+-5.0$ & 0.93 \\
\hline $\begin{array}{l}\text { Previous treatment } \\
\text { with }>2 \text { IS }\end{array}$ & $4(40 \%)$ & $7(43.8 \%)$ & 1.000 \\
\hline Corticosteroids & $7(70 \%)$ & $10(62.5 \%)$ & 1.000 \\
\hline Hydroxychloroquine & $1(10 \%)$ & $5(31.3 \%)$ & 0.352 \\
\hline Azathioprine & $2(20 \%)$ & $2(12.5 \%)$ & 0.652 \\
\hline Mycophenolate & $1(10 \%)$ & $1(6.3 \%)$ & 1.000 \\
\hline $\begin{array}{l}\text { Corticosteroids with } \\
\text { other } \\
\text { immunosuppressive } \\
\text { agents }\end{array}$ & $4(40 \%)$ & $7(43.8 \%)$ & 1.000 \\
\hline Mortality & $2(20 \%)$ & $1(6.3 \%)$ & 0.538 \\
\hline $\begin{array}{l}\text { Haematological } \\
\text { disease }\end{array}$ & $7(70 \%)$ & $10(62.5 \%)$ & 1.000 \\
\hline Renal impairment & $3(30 \%)$ & $8(50 \%)$ & 0.425 \\
\hline CNS disorders & $3(30 \%)$ & $3(18.5 \%)$ & 0.644 \\
\hline Psychosis & $1(10 \%)$ & $0(0 \%)$ & NA \\
\hline Serositis & $0(0 \%)$ & $1(6.3 \%)$ & NA \\
\hline Mesenteric vasculitis & $1(10 \%)$ & $2(12.5 \%)$ & 1.000 \\
\hline Livedo reticularis & $3(30 \%)$ & $3(18.8 \%)$ & 0.644 \\
\hline Joint damage & $7(70 \%)$ & $15(93.8 \%)$ & 0.264 \\
\hline Skin damage & $9(90 \%)$ & $13(81.3 \%)$ & 1.000 \\
\hline Lung injury & $1(10 \%)$ & $0(0 \%)$ & NA \\
\hline $\mathrm{PH}$ & $1(10 \%)$ & $1(6.3 \%)$ & 1.000 \\
\hline
\end{tabular}

Table 11. Characteristics of patients with SLE and HCV with/without post IS (immunosuppression) HCV reactivation ${ }^{(18)}$

CNS - central nervous system; PH - pulmonary hypertension. 
Taking into consideration that they are recently introduced in the antiviral treatment regimen, the safety of DAAs in patients with chronic kidney disease is under continuous evaluation.

Studies conducted on patients with lupus showed that although discontinuation of immunosuppression during DAAs treatment due to fear of possible interactions between the 2 treatment categories, proteinuria and inflammatory markers decreased significantly from week 4 of antiviral therapy, especially in patients who previously have had a response to immunosuppression. Moreover, patients are able to reach $\mathrm{SVR}^{(20)}$.

In case of lupus nephritis, patients are at high risk of developing chronic end-stage kidney disease, with an estimated incidence of $15 \%$, and the association with $\mathrm{C}$ virus infection increases the progression rate to this type of pathology, decreases the survival rate of patients.

Mitwalli et al. included 134 patients with class IV lupus nephritis with/without $C$ virus infection in the study, followed up between 1995-2008 over a period of $6.7 \pm 3.3$ (1-14.4) years.

Patients received the same immunosuppression regimen (corticotherapy and cyclophosphamide), without antiviral treatment. Higher proteinuria was found in patients with $\mathrm{C}$ virus infection, but similar immunological profile and liver function.
Furthermore, patients with lupus nephritis and $C$ virus infection presented a faster deterioration of kidney function towards stage 5 CKD, with an interval of $95 \%$ and $80 \%$ until the initiation of dialysis, for the group without $\mathrm{C}$ virus infection and of $90 \%$ and $65 \%$ for the group with $C$ virus infection at 5 and 10 years of follow-up, respectively ${ }^{(21)}$ (Figure 1, Figure 2, Figure 3).

\section{Conclusions}

The increased prevalence of $C$ virus infection in SLE patients advocates the hypothesis that $C$ virus is a potential triggering agent of SLE. However, for diagnosis the clinician should take into consideration the 3 situations: False positive SLE and Anti-HCV Ab, confirmed SLE and positive Anti-HCV Ab, C virus infection mimicking SLE via the presence of specific antibodies and symptomatology. In terms of treatment, on the one hand immunosuppression influences viral replication, on the other hand antiviral therapy itself may trigger lupus-like diseases or even SLE.

Obviously, the prognosis of patients is poor, having a faster evolution towards chronic endstage kidney disease and a higher mortality than the general population. This category of patients therefore need a personalized diagnosis, treatment and follow-up, taking into account also the particularities of each patient. 


\section{Refferences}

1. Hepatitis $C$ virus infection mimicking systemic lupus erythematosus - study of hepatitis $C$ virus infection in a series of 134 spanish patients with systemic lupus erythematosus, Ramos-Casals M. Et al, Arthritis \& Rheumatism, vol 43, no 12, 2000; pp 2801-2806.

2. Systemic autoimmune diseases co-existing with chronic hepatitis $C$ virus infection (the HISPAMEC Registry): patterns of clinical and immunological expression in 180 cases. Ramos-Casals M et al, Journal of Internal Medicine 2005; 257:549-557.

3. Prevalence of Active Hepatitis C Virus Infection in Patients with systemic lupus erhythematosus, Mubashir et al., American Journal of Science, 2006; 331(5):252256.

4. Hepatitis C virus antibodies in systemic lupus erythematosus, Kowdley KV et al, J Clin Gastroenterol 1997, Sep 25(2):437-9

5. Hepatitis $C$ virus infection in systemic lupus erhytematosus: a case-control study, Perlemuter et al, J Rheumatol 2003; 30;1473-1478.

6. Hepatitis $C$-associated glomerulonephritis mimicking systemic lupus erythematosus, Krajewska $M$ et al, Scandinavian Journal of Rheumatology,2015, At:22:41.

7. Delayed onset of systemic lupus erythematosus in patients with "full-house" nephropathy, Gianviti A. et al., Pediatr Nephrol. 1999; 13(8):683-7.

8. Lupus-like glomerulonephritis: an autoimmune complication of hepatitis C infection, Hobeika L et al, CEN Case Rep (2012) 1:29-33.

9. Low titre auto-antibodies predict autoimmune disease during interferon-alpha treatment of chronic hepatitis C, Bell T.M. et al, J Gastroenterol Hepatol, 1999; 14(5):419-22.

10. Interferon alpha-induced lupus Proof of Principle, Niewold T. J Clin Rheumatol, 2008 ; 14(3): 131-132.

11. Hepatitis $C$ virus infection, interferon alfa and lupus; a curious combination, Martinez-Mier $G$ et al, Nefrologia 2014; 34(4):534-6.

12. Severe systemic lupus erythematosus induced by antiviral treatment for hepatitis C, Ho V. et al, J Clin Rheumatol 2008;14:166-168.

13. Lupus-like immune complex-mediated glomerulonephritis in patients with hepatitis $C$ virus $C$ infection treated with oral, interferon- free, direct acting antiviral Sise et al, Kidney Int Rep (2016) 1, 135-143.

14. Reactivation of replication of hepatitis $B$ and $C$ viruses after immunosuppressive therapy: an unresolved issue, Vento et al, Lancet Oncol 2002; 3:333-40.

15. Reactivation of hepatitis $C$ virus and its cllinical outcomes in patients treated with systemic chemotherapy or immunosuppressive therapy, Him Lee et al, Gut and Liver, vol 11, No 6, 2017, pp 870-877.

16. Hepatitis $C$ virus infection and vasculitis - implication of antiviral and immunosuppressive therapies, Vassilopoulos et al, Arthritis \& Rheumatism, vol 46, no 3, 2002, pp 585-597

17. Treatment of hepatitis $C$ in patients undergoing immunosuppressive drug therapy, Ooka $\mathrm{K}$ et al, Journal of Clinical and translational hepatology, 2016 vol 4; 206-227. 18. Incidence and antiviral response of hepatitis $C$ virus reactivation in lupus patients undergoing immunosuppressive therapy, Chen $\mathrm{W}-\mathrm{H}$. et al, Lupus (2015), http://lup.sagepub.com, 2015.

19. Association of hepatitis $C$ seropositivity with increased risk for developing end-stage renal disease, Tsuijl et al, Arch Intern Med 167: 1271-1276.

20. Safety and efficacy of direct-acting antivirals for chronic hepatitis $C$ in patients with chronic kidney disease, Iliescu L. Et al, BMC Nephrology (2020) 21:21.

21. Effects of concomitant hepatitis $C$ virus infection in patients with underlying lupus nephritis on long-term renal outcome, Mitwalli $H$ et al, Nephrol Dial Transplant (2012) 27:627-632 